\title{
Split-leg percutaneous nephrolithotomy: A safe and versatile technique
}

\author{
Eugenio Di Grazia, Pasquale La Rosa \\ U.O.C. Urologia-ARNAS Garibaldi, Catania, Italy.
}

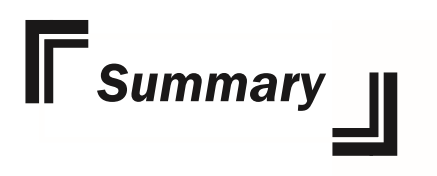

Objectives: Percutaneos nephrolithotomy (PCNL) is the gold standard for treatment of urinary stones larger than $2 \mathrm{~cm}$ and refractory to ESWL. Nowadays most debate about surgical technique is related to the positioning of patients. We report our experience on prone PCNL with split-leg variant (SL-PCNL) Materials and methods: 30 consecutive patients underwent prone SL-PCNL. Preoperative stone size was determined by measuring stones longest diameter on CT scan. In cases with multiple stones, stone size was determined by the sum of each stone diameter on CT scan. Patients evaluated consisted of 20 females and 10 males and median age was 55 (20-72). The average BMI was 27 (24-35). 15 patients had multiple stones, 10 pyelocalicial, 10 pelvic larger than $2 \mathrm{~cm}, 2$ in horseshoe kidneys and 3 staghorn stones.

Results: Stone free rate was $87 \%$ after first look and 97\% after second look. In 2 cases, we used a flexible ureteroscopy $7.5 \mathrm{Fr}$ (Flex 2 - Storz) to treat a calculus in ureter or for a contemporary double access (Endoscopic combined Retrograde Intrarenal Surgery ECIRS). In 28 cases we placed a 20 fr nephrostomy while in two cases procedure was tubeless. In 20 cases we placed a double-J catheter. In 2 cases we performed two tract and in 2 horseshoe kidneys access was close to spine. The average surgical time was about 90 minutes (range 30-120 minutes). Hemoglobin drop was about $1.5 \mathrm{mg} / \mathrm{dl}$ (range 1-3 .4 mg/dl) and no major complications were reported.

Conclusions: In our experience PCNL in prone with spread-legs variant is a versatile technique and allows to match the advantages you have with same technique in supine, providing at the same time benefits in cases of anatomical abnormalities, challenging cases, or when multi-tract accesses are required.

KEY WORDS: PCNL; Percutaneous nephrolithotomy; Urinary stones; Prone; Supine.

\section{INTRODUCTION}

Percutaneous nephrolithotomy is the gold standard in treating kidney stones larger than $2 \mathrm{~cm}$ (1). The technique has been modified and customized by many endourologists since its introduction in 1976 by Fernstrom and Johanson. Many various safe and effective changes in patient positioning for PCNL have been proposed over years, including reverse lithotomy position (2), prone split-leg position (3-4), lateral decubitus $(5,6)$, supine position (7), and Galdakao-modified supine Valdivia (GMSV) position (8). In recent years it has been observed a remarkable increase in performing PCNL in supine decubitus, although others have remained faithful to prone technique because supine decubitus doesn't seem to provide great benefits for morbidity and effectiveness (9), otherwise the prone position provides a larger area for the percutaneous renal access, a wider space for instrument manipulation, and a presumed lower risk of splanchnic injury. Recently we adopted a variant to our technique in prone position, by splitting legs allowing surgeons a dual approach through retrograde and anterograde paths whithout changing decubitus. Aim of this study is to test safety, advantages and feasibility of this technique. 


\section{Materials AND METHOdS}

After a series of about 300 patients undergoing PCNL from 2002 to 2012, we evaluated 30 consecutive cases performed in prone decubitus with split-leg variant (SLPCNL). Preoperative evaluation included history, clinical examination and basic laboratory investigations.

Radiological investigations included pelvi-abdominal ultrasonography and computerized tomography (CT) for all patients. Preoperative stone size was determined by measuring stones longest diameter on CT scan. In cases with multiple stones, stone size was determined by the sum of each stone diameter on CT scan. Patients evaluated consisted of 20 females and 10 males and median age was 55 (20-72). The average BMI was about 27 (2435 BMI). 15 patients had multiple stones, 10 pyelocalicial, 10 pelvic larger than $2 \mathrm{~cm}, 2$ in horseshoe kidneys and 3 staghorn stones (Table 1). We practiced an antibiotic prophylaxis the evening before the procedure with a cephalosporin of III generation in case of sterile urine culture. When culture was positive generally we started a targeted antibiotic therapy a week earlier.

Table 1.

Stone characteristics.

\begin{tabular}{|ll|}
\hline Site & 15 multiple \\
& 10 pyelocalicial \\
& 5 pyelic \\
& 3 staghorn; 2 horseshoe kidney \\
\hline Associated ureteral stones & 2 cases \\
\hline Accesses & 28 single accesses \\
& 2 double accesses \\
& 2 upper calix accesses in \\
horseshoe kidney
\end{tabular}

Figure 1.

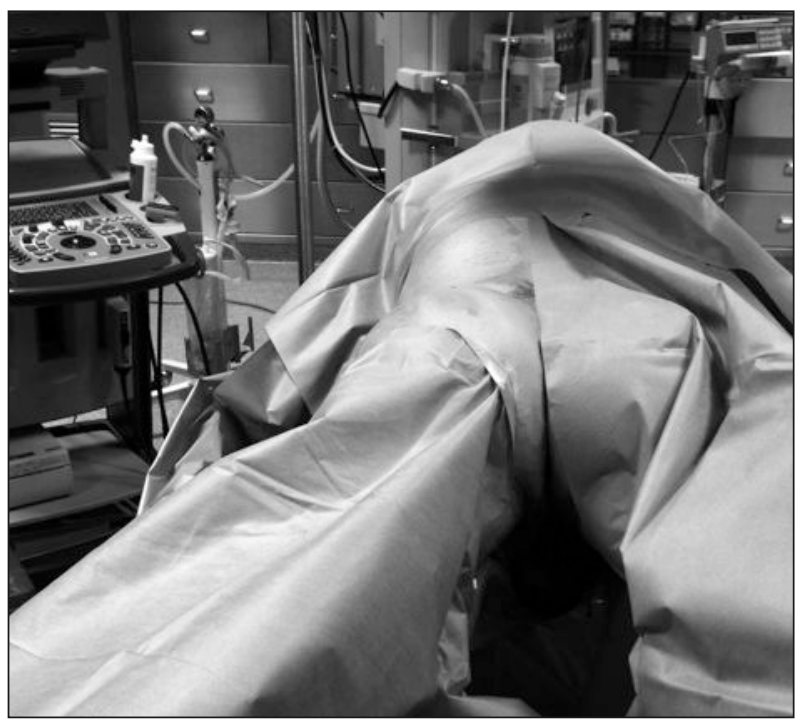

Figure 2.

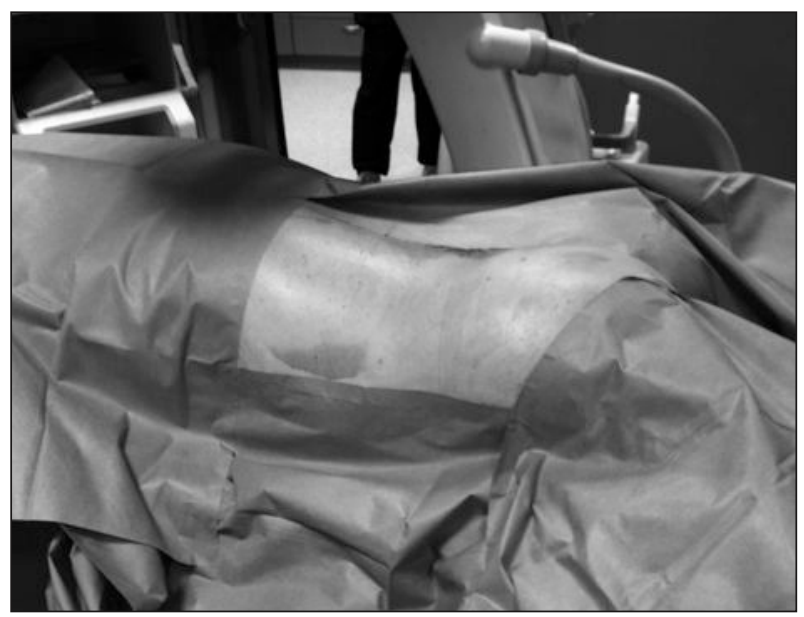

\section{Procedure}

After general anesthesia patient was placed in prone position with legs apart (Figures 1-2). With a 15 F flexible cystoscope (Stors) under fluoroscopic guidance (C-arm) a $5 \mathrm{~F}$ open-end ureteral catheter was positioned until renal pelvis for contrast dye injection during percutaneous access; in 2 cases, we used a flexible ureteroscopy $7.5 \mathrm{Fr}$ (Flex 2 -Storz) to treat a calculus in ureter or for a combined access Endoscopic Combined Intrarenal Surgery (ECIRS). The percutaneous kidney access is performed by combined echo-radiological approach and tract is dilated with balloon to place a $24 \mathrm{~F}$ Amplatz sheath (X force-Bard). The litotripsy was accomplished by a $24 \mathrm{~F}$ rigid nefroscope or $15 \mathrm{~F}$ flexible nefroscope (Storz) using ultrasound energy sources (Storz), ballistic (EMS) and Laser (Dornier). Operative time was determined by estimating the time from the application of the ureteric catheter to the placement of the nephrostomy tube. At the end of procedure we usually place a $20 \mathrm{~F}$ nephrostomy or ureteral stent according to the degree of bleeding or of stone clearence.

\section{RESULTS}

All cases were punctured successfully. Stone free rate was $87 \%$ after first look and $97 \%$ after second look (Table 2). At the end of procedure in 28 cases we placed a $20 \mathrm{~F}$ nephrostomy while in two case the procedure was tube-

Table 2.

Success rate and complications.

\begin{tabular}{|ll|}
\hline Success rate & First look (87\%) \\
& Second look (97\%) \\
\hline Complications & Bleeding requiring transfusion in 1 (3,3\%) \\
& Persistent fever over 38,5 $\mathrm{C}^{\circ}$ in $4(13,3 \%)$ \\
& Prolonged urinary leakege in 2 (6,6\%) \\
\hline
\end{tabular}


less. In 20 cases we placed a double-J catheter. The nephrostomy was retrieved when any bleeding ceased and when no residual fragments were demonstrated at post-surgery radiological assessment. In those cases with residual fragments we performed a second look with flexible nefroscope, small baskets and Holmium Laser lithotripsy accessing the same matured tract without Amplatz sheath.

When $20 \mathrm{~F}$ nephrostomy is withdraw the tract is large and mature enough to allow access of $15 \mathrm{~F}$ flexible nephroscope through the kidney without patient discomfort as performed in an outpatient procedure.

In 2 cases we performed two tract to clear stones and in 2 horseshoe kidneys target calix was close to the spine. The average surgical time was about 90 minutes (range 30-120 minutes)

The drop in hemoglobin was about $1.5 \mathrm{mg} / \mathrm{dl}$ (range 13.4). Applying classification of Clavien-Dindo in 1 case (3.3\%) we practiced a transfusion of a blood unit (grade II). 4 patients (13.3\%) had a persistent fever over $38.5^{\circ}$ C for more than 2 days (grade II). In 2 cases $(6,6 \%)$ was necessary to reposition the stent for persistent urinay leakage (grade II). No case of visceral perforation or other major complications were reported.

\section{Discussion}

The SLPCNL is a technique already presented in literature by Grasso et al. with aim of facilitating both contemporary antegrade and retrograde approach to upper urinary tract. In their experience Grasso et al. reported a $41 \%$ of cases where this position was useful for dual instrumentation (3).

Also Scarpa et al. described this approach in solving a ureteral stenosis with combined antegrade and retrograde approach (4).

Many studies have now demonstrated equivalence of PCNL in supine and prone positions, however proponents of supine believe that it offers advantages over the prone: no repositioning, lack of patient handling, spontaneous gravitational fall of fragments, less time consuming because of not repositioning, greater comfort for surgeon, reduced x-ray exposure, low pyelic pressures, retro and antegrade access simultaneously (8-9-10). Nevertheless as seen by our experience SLPCNL provides as many benefits as supine. Many endourologists performing PCNL in prone position place a ureteral catheter for injecting contrast dye in supine decubitus, then they reposition patient in prone to start their percutanous procedure

A real criticism can be that patient repositioning is timeconsuming and associated with patient discomfort, increases radiological hazard to urologist's hands, and asks for several nurses to be present for intraoperative changes of decubitus in case of simultaneous retrograde instrumentation of ureter (implying evident risks related to pressure points and possible ocular, spinal, or peripheral nerve injuries).

In our experience it should be correct to talk of "positioning" patient in prone position, rather than "repositioning", as patient is gently rotated from a stretcher to operating bed without spending much time and neces- sitating extra nurses. After draping, we start the procedure in prone and upper urinary tract may be contemporary instrumented both in an antegrade and retrograde fashion by two surgeons. The main difference between prone and supine is the impossibility for surgeon using flexible scopes to access upper urinary tract in prone while both semirigid or flexible instruments can be used in the latter.

Another criticism over prone position rise up when obese patients or patients affected by respiratory diseases are concerned, or in cases where extensive controlateral or omolateral ureteral instrumentation is requested before or contemporary to percutaneous access. CROES studies demonstrated no significant differences in complications between prone and supine when such patients are concerned (11).

In our opinion further experience on using flexible ureteroscope in split-leg position may overwhelm these presumed obstacles in traditional prone position. If no gross disadvantages are reported between prone and supine, we assert benefits that prone decubitus can account for: easier way of perform mutiple tracts when is necessary, greater freedom of movement of instrumentation. In addition, upper-pole calyx calyceal puncture is quite challenging as upper pole is normally more medial and posterior and concealed deeply in the rib cage, when patient is positioned supine (12). Prone position account for an easier access to horseshoe kidney as target calix is normally close to spine, rendering access in supine very challenging.

This is a description of technique with no direct comparison with a homogeneous control population treated in others surgical positions, however the advantage we obtained by adopting this variant to the traditional prone position makes us to propose such technique as a useful option for percutaneous renal surgery.

\section{Conclusions}

In our experience PCNL in prone with spread-legs variant is a versatile technique that allows to match the advantages you have with same technique in supine, providing at the same time benefits in cases of anatomical abnormalities, challenging cases, or when multi-tract accesses are required.

\section{References}

1. Fernstrom S, Johansson B. Percutaneous pyelolithotomy. A new extraction technique. Scand J Nephrol Urol. 1976; 10:257-9.

2. Lehman T, Bagley DH. Reverse lithotomy, modified prone position for simultaneous nephroscopic and ureteroscopicprocedures in women. Urology. 1988; 32:529-31.

3. Grasso M, Nord R, Bagley DH. Prone split leg and flank roll positioning antegrade and retrograde: simultaneous access to the upper urinary tract. J Endourol. 1993; 7:307.

4. Scarpa RM, Cossu FM, De Lisa, et al. Severe recurrent ureteral stricture: the combined use of an anterograde and retrograde approach in the prone split-leg position without x-rays. Eur Urol. 1997; 31:254-6

5. Kerbl K, Clayman RV, Chandhoke PS, et al. Percutaneous- 
stone removal with the patient in a flank position. J Urol. 1994; 151:686-8

6. Gofrit ON, Shapiro A, Donchin A, et al. Lateral decubitusposition for percutaneous nephrolithotripsy in the morbidlyobese or kyphotic patient. J Endourol. 2002; 16:383-386.

7. Uria in Valdivia JG, Valle Gerhold J, Lopez JA, et al. Technique and complications of percutaneous nephroscopy: experience with 557 patients in the supine position. J Urol. 1998; 160:1975-8.

8. Scoffone CM, Cracco CM, Cossu M, et al. Endoscopic combined intrarenal surgery in Galdakao-modified supine Valdivia position: a new standard for percutaneous nephrolithotomy? Eur Urol. 2008; 54:1393-403.

9. Valdivia JG, Scarpa RM, Duvdevani M, et al. Croes PCNL Study
Group. Supine versus prone position during percutaneous nephrolithotomy: a report from the clinical research office of the endourological society percutaneous nephrolithotomy global study. J Endourol. 2011; 25:1619-25

10. Autorino R, Giannarini G. Prone or Supine: Is This the Question? Eur Urol. 2008; 54:1216-1218.

11. Fuller A, Razvi H, Denstedt JD, et al. CROES PCNL Study Group. The CROES percutaneous nephrolithotomy global study: the influence of body mass index on outcome. J Urol. 2012; 188:138-44.

12. de la Rosette JJ, Tsakiris P, Ferrandino MN, et al. Beyond prone position in percutaneous nephrolithotomy: a comprehensive review. Eur Urol. 2008; 54:1262-9.

\section{Correspondence}

Eugenio Di Grazia, MD (Corresponding Author)

Via Galermo 171/c - 95123 Catania, Italy

eugeniodigrazia@hotmail.com

La Rosa Pasquale, MD

Via Palermo 636 - 95100 Catania, Italy

pasq.larosa@alice.it 